\title{
Collaboration in Commissioned Research: Benefits and Challenges of Scholar/Practitioner Partnerships in Conflict Contexts
}

Words: 2948

Submission to PS Symposium

\section{Introduction}

Commissioned research is research paid or funded by a third party with contracting agents ranging from governmental bodies, non-governmental organizations (NGOs), and private companies. Commissioned research presents ethical issues to the researchers where funding agents will often instrumentalize the results to prove the legitimacy of programs (Ham 1999). Additionally, commissioned research also challenges researchers as they work to quickly produce reports based on an agreed timeline, undermining abductive reasoning and the quality of findings (Richter \& Hostettler 2015).

In this article, we discuss how collaborative methodologies, through the creation of strong collaboration between scholars and practitioners in conflict contexts, can help bridge the scholar-practitioner gap, merging rigorous and ethical political science research with real-world problem-solving.

As Firchow and Gellman explain in the introduction to this symposium, collaborative methodologies center people as stakeholders and allies in research designs, enabling researchers and everyday citizens to work together, rather than the "expert" researcher descending upon a community for conventional data extraction. Further, collaborative methodologies naturally prioritize "real-world" impact in addition to filling gaps in the academic literature. These benefits can enhance many forms of political science research, as this symposium illuminates. However, these attributes have been especially crucial in our commissioned research in conflict contexts, first in providing pathways for both scholarly and pragmatic outputs, and second for facilitating more equitable and ethical research dynamics that are often encountered in contracted research.

This article is organized as follows: first, we explain the context and positionality of our research, and how we use collaborative methodologies our work. Second, we describe the challenge of bridging scholar-practitioner divides in commissioned research, and we discuss how collaborative methodologies can ameliorate some of those challenges, facilitating more rigorous research outputs and ethical practices. Third, we acknowledge remaining obstacles and offer best practices for political scientists employing collaborative methodologies in commissioned research.

\section{Context and Positionality}

Our discussion is guided by our experiences as UK-based political scientists conducting multiple international non-governmental organization (INGO) commissioned research projects on conflict and resilience in central Africa, east Asia, and the Middle East. Specifically, we draw from an ongoing European Union (EU) funded research consultancy with an INGO, assessing the integration of their humanitarian programs to strengthen community resilience to violence (otherwise known as conflict sensitivity), using the case studies of Burundi, the Democratic Republic of the Congo (DRC), Myanmar, and South Sudan. Our collaboration with practitioners in the fieldwork crosses three main levels: collaboration with the NGO national office staff in each of the countries; collaboration with the local partner organizations who administer the NGO's programs in each country; and collaboration with the local communities in each country who are the beneficiaries of (and participants in) the NGO's projects. Our collaboration is 
primarily with NGO staff in formulating the research questions and research design, with local partners in piloting the methodology, with local partners and community members in data collection, and with NGO staff and local partners in dissemination.

We are aware of the issues of power and positionality between ourselves and our collaborators, and also disparities among and between our local partners. For ourselves, we are both white-presenting, US/Europe-educated researchers, working in these projects as consultants of a major international NGO, so we are mindful of the privilege we have and the perceptions that some partners may have of us in these collaborations. Thus, even as we strive for equitable relationships, we are aware of the inherent asymmetries in all research, but especially in NGOcommissioned research where we are seen not just as scholars, but as representatives of a donor. This positionality intrinsically links researchers to the development institutions and marks them as potential funding agents, endangering the opportunity to create meaningful partnerships.

The creation of partnerships themselves is not problem-free and may create or reinforce local asymmetries in communities where we work. For example, while we work with practitioners at multiple levels (local, national, regional and international), it is clear that partners at the national NGO level have a certain degree of power relative to the local organizations with whom they partner, and those partners in turn have a degree of power in the communities they serve. This positionality is something that all researchers should keep in mind, especially when relying on local interlocutors to act as gatekeepers to communities, but it is especially pronounced in NGO-commissioned research where researchers are more reliant on the networks of the funding organizations.

\section{Bridging Scholar-Practitioner Divides via Collaborative Methodologies}

Commissioned research often asks political scientists to provide narrow, project-based findings. As a result, researchers may find themselves epistemologically split between focusing on a 'problem-solving' approach, prioritizing pragmatic, program-focused recommendations, and a scholarly approach, oriented around theoretical and conceptual contributions. In other words, a key challenge of commissioned research is that NGOs often seek research to assess a particular aspect of a program or project, which makes it challenging to construct a research design that can fulfils the client's requirements (and hopefully improves the program), while informing knowledge beyond the narrow confines of the immediate project. To compound this, NGOs employ metrics by which they measure their work using language which is based on internal organizational priorities and sector jargon, making it challenging to translate academic understandings of issues to fit into the needs of the NGO and vice versa.

In our work, we have found that collaborative methodologies with community partners can help ameliorate some of these scholar-practitioner divides. First, in terms of research design, we work closely with local practitioners at multiple levels to gain an understanding of the issues facing their communities. First, via informal interviews and small-group conversations, we listen as local partners outline their experience of working in the community and what they believe are the key challenges. Aware that the particular positionality of partners in communities will impact what they find valuable to tell us, we then paint a larger picture by triangulating findings with knowledge from focus group discussions, interviews, and community mapping with community members. These conversations with local partners and beneficiaries allow us to then work with NGO staff to refine research questions and decide together on appropriate methodologies, which we then pilot together. This collaborative process helps bridge the gap between researchers and partners by ensuring that both the research questions and the methodologies are useful and 
applicable to all participants. Furthermore, it enhances the rigor of our research by allowing us to more fully develop our research questions and include more multi-level analysis in our research design.

Second, we also work in collaboration with practitioners to ameliorate the linguistic and conceptual challenges, as commonly understood terms in the NGO sector do not always translate smoothly in communities or in academia. For example, we charted the different interpretations of the word 'conflict' from the EU donor, to the international office of the NGO, the regional offices, to the local partners who interpreted it at the grassroots level. Through this process the word was translated multiple times, with understanding varying between the international, regional and local levels. The variance of understanding had a significant impact on the implementation of the project and sometimes resulted in friction between international and grassroots actors. Placing participants at the fulcrum of the research and methodology allowed us to emphasize the perceptions of the participants in the study and use their context-specific definitions that were originally conceptualized in more abstract terms by the NGOs. This process helped us develop generalizable terms which extended across different cases, and amplified local understandings that extended beyond the parameters of the original NGO program design. As Lauren MacLean discusses in this volume, working collaboratively can strengthen conceptualization both within and between projects.

Third, we work in collaboration with practitioners in disseminating our research findings. One problem we have observed between commissioned researchers and practitioners is that the final reports that clients have commissioned do not always help forward local partners' understanding of the issues that affect them. The primary reason for this is that dissemination of findings normally takes place far away from the conflict context in the safety of universities or NGO offices, and normally no follow-up activities are conducted within field sites to transfer knowledge more widely. We avoid this problem by sharing early drafts of reports with local partners to elicit feedback and facilitate discussion, ensuring that our findings are seen by the partners and we can discuss further details with them directly. An example of good practice was when the commissioning NGO on our project invited the relevant local staff to one of the European offices to participate in a workshop with us to discuss the lessons learnt from our research and possible methods to implement recommendations. In addition, we collaborate with the commissioner of the research through country and head offices to identify not only international areas of dissemination, but local, and regional as well. Furthermore, the collaborative approach has also helped us foster a 'do no harm' approach in dissemination, communicating directly with our partners before sharing findings to ensure that the safety of colleagues and beneficiaries is not compromised.

\section{Enhancing Equitable and Ethical Practice via Collaborative Methodologies}

Collaborative methods not only help bridge scholar-practitioner divides, but also power asymmetries and "Global North/South" lines, as discussed in the 2018 PS: Political Science and Politics Profession Symposium on community engaged research methods (Michelitch, 2018; Bleck, Dendere, and Sangare, 2018). In our research, we aim to avoid the "North/South" dichotomy, and instead focus on deconstructing elitist approaches to research that fail to take into account the agency of people in communities being studied. This is especially crucial when conducting commissioned research in the humanitarian sector, where aid and development are often criticized as being neo-colonialist by reinforcing structures of power and dependency. 
We find that collaborative methodologies, though not eradicating power asymmetries, can help reduce the disparity of power between commissioning agents and grassroots actors. One clear problem for researchers in commissioned research projects is that there is a palpable fear among local partners that critiquing programs being implemented by the contracting NGO will lead to funding being discontinued. Local partners resultingly try during field visits of the NGOs and researchers to put 'the best foot forward', highlighting successes and glossing over issues they might have encountered. As will be explained in the next section, due to the fragile and conflict dynamic of the places in which we work, we faced and overcame implicit pressure to try and put more positive spin in final reports to better reflect our partners.

Collaborative methods reduces both impulses by contextualizing the the positionality of the researchers in the eyes of collaborators, not as agents of the funders, but as colleagues who can help them address problems they face. To do this, we spend significant time informally with the local partners and engaging with them during our research design phase, ensuring that we integrate local partners insights into the design, and making them aware that their prescriptive is valued and crucial to research. Local partners are moved beyond participants of the study and are now stakeholders, a relationship that is further developed through recruiting the local partners to comment on early drafts of the reports. By establishing direct relationships that are not managed by the commissioning NGO, local partners are able to speak freely on issues with programming. The result is that the research is now able to comment on potentially harmful dynamics of development programming without imperiling the local partners' position with the funding agent. This is a small, meaningful step to reduce power asymmetry between NGOs and grassroots partners and places local insight and at the center of reports, filtering their knowledge directly back to donor community.

\section{Challenges and Best Practices}

While our experience with collaborative methodologies has been positive overall, it is not without its challenges. As Jennifer Cyr discusses in this symposium, there are potential pitfalls in trying to do too much when blending voices, especially when using mixed methods, as we often do in our research. Further, as Mneesha Gellman points out in her article, collaborative methodologies also bring logistical challenges, and are often more time and labor intensive than a more traditional approach.

In commissioned research, and specifically in the humanitarian sector in which we work, the biggest challenge in collaborative research with practitioners is maintaining neutrality. We agree with Oliver Kaplan who argues in this symposium that the partial independence of analysis can enhance the credibility, as well as the activist power, of findings. We have found the same in our work, and maintain some independence in our data analysis, doing our own coding of interviews and focus groups and drawing preliminary conclusions before sharing and discussing with collaborating practitioners. As noted above, our research is often intertwined with aid and development programs; the fact that our collaborators are often practitioners or beneficiaries within those programs makes it necessary to maintain some independence in this part of the research process.

The question of neutrality raises other issues in commissioned research. Since we work in 'fragile contexts,' or places in which violent conflict intersects with humanitarian needs, we are mindful that our findings will likely inform NGO program design and donor investment in positive or negative ways with real life consequences for communities living in dire contexts. 
Further, even though we are not evaluators, the simple fact that we do collaborate with NGO staff for much of the research process made us initially anxious to communicate critical findings.

However, we found that staying objective was not only valued by us as researchers, but also by our practitioner collaborators. First, in working closely with local communities themselves, it underscored the fact that drawing conclusions that were anything less than objective would ultimately hurt those communities in the long term, as it was necessary to communicate critical findings to improve programs that required certain changes. Furthermore, the fact that we developed collegial relationships with country staff and local partners actually allowed us to have more critical, self-reflexive conversations with them about what was working and what wasn't, a level of depth that we would not have gotten through a singular extractive interview. These conversations revealed difficulties and frustrations within the NGO programs that were not always visible on the surface, and encouraged us to take a more critical eye as well.

The question around objectivity applies not just to our practitioner-focused outputs, but our academic ones also, and the previously mentioned challenge of bridging the practitionerscholar gap in commissioned research. This tension has not only methodological implications, but ontological as well, especially for political scientists working from a critical theory orientation. Critical theorists argue that problem-solving based research (focused on pragmatic, policy-oriented findings) that is typical in commissioned research does not sufficiently take into account underlying structural issues (Pugh 2004), while those who take the problem-solving approach argue that critical theories do not provide timely or workable solutions to immediate problems. This tension is especially present in the humanitarian aid sector, where critical theorists often development organizations as perpetuating dependency, or even being unintentionally complicit in government agendas. This was the case in Myanmar for example, where aid agencies' support for Internally Displaced Persons (IDP) camps essentially enabled the government's forced removal and isolation policy around the Rohingya minority, although withdrawing support would have essentially meant the end of those communities. We believe it is vital for researchers to deliver on commissioned research to NGO clients that, in the problemsolving tradition, helps address real world problems, while also being able to analyze the broader structural issues undergirding the sectors or contexts in which they work. This is a fine balance to strike, but in dealing with this tension deliberately, researchers can ensure that the work they produce on commissioned projects can make meaningful scholarly contributions (that the data collected can potentially contribute to peer-review) while providing useful recommendations to a client.

\section{Conclusion}

The challenges we have outlined are not unique to commissioned research, nor to research in conflict areas. However, throughout our work we have seen how the particular nature of commissioned research can limit researcher independence and undermine the agency of local partners if not pursued collaboratively. Furthermore, the problem-solving approach to data collection in commissioned research can prevent researchers from tackling more 'puzzle-based' questions, such that empirical findings are often difficult to translate into theory-developing research articles. Yet collaborative approaches that lighten the political scientist's footprint in observable ways need not make it harder for the conversion of data collected into theory development work. Indeed, rather than giving up on this goal, scholars should continue to engage in commissioned research, but adopt practices that consider how researchers should work as well as what is collected. In our experience, engaging local practitioners as partners in such research, 
especially in conflict zones, can allow for enhanced two-way knowledge exchange throughout the process, from the development of the research design, through the data collection, and to the dissemination, facilitating more ethical research practices and methodologically rigorous results.

\section{Works Cited}

Bleck, Jaimie, Dendere, Chipo, \& Sangaré, Boukary. (2018). Making North-South Research Collaborations Work. PS: Political Science and Politics, 51(3), 554-558.

Ham, Vince. (1999). Tracking the truth or selling one's soul? Reflections on the ethics of a piece of commissioned research. British Journal of Educational Studies, 47(3), 275-28

Michelitch, Kristin. (2018). Whose Research Is It? Political Scientists Discuss Whether, How, and Why We Should Involve the Communities We Study. PS: Political Science \& Politics, 51(3), 543-545.

Pugh, Michael. (2004). Peacekeeping and critical theory. International peacekeeping 11(1), 3958.

Richter, Marina, and Ueli Hostettler. (2015) "Conducting commissioned research in neoliberal academia: The conditions evaluations impose on research practice." Current sociology, 63 (4), 493-510 